these remission criteria to categorize $51.1 \%$ of the children as having persistent JIA (active disease for >24 months), $6.7 \%$ as having polycyclic JIA (alternating active and inactive disease), and $42.2 \%$ as having monocyclic JIA (active disease for $<24$ months).

The presence of joint inflammation, fever, or an erythrocyte sedimentation rate $>26 \mathrm{~mm} / \mathrm{h}$ at 3-month follow-up, and corticosteroid use or an erythrocyte sedimentation rate $>26 \mathrm{~mm} / \mathrm{h}$ at 6-month follow-up, was associated with polycyclic or persistent JIA, and increased time to remission.

The authors suggest that identifying those at risk of developing persistent JIA could allow these patients to be offered more-aggressive treatments.

Original article Singh-Grewal D et al. (2006) Predictors of disease course and remission in systemic juvenile idiopathic arthritis. Arthritis Rheum 54: 1595-1601

\section{Economic analysis of gastroprotective treatments in patients with chronic arthritis}

Researchers from England have conducted an economic analysis to establish which gastroprotective treatments are cost-effective in patients with chronic arthritis who regularly take nonsteroidal anti-inflammatory drugs (NSAIDs). New drugs, such as the cyclo-oxygenase 2 (COX2)-selective inhibitors (coxibs) generally cost more than existing drugs; however, claims of improved safety and efficacy for new drugs can prematurely reduce the use of existing drugs.

Data from a previously published systematic review, which included 74,666 patients with chronic arthritis from 112 randomized, controlled trials of gastroprotective treatments, were combined with up-to-date UK resource-use and unit-cost data.

The authors ranked the available treatments from least safe to most safe, with respect to gastrointestinal toxicity: NSAIDs alone; COX2-preferential inhibitors; NSAIDs plus $\mathrm{H}_{2}$-receptor antagonists $\left(\mathrm{H}_{2} \mathrm{RAs}\right)$; coxibs; NSAIDs plus misoprostol; and NSAIDs plus proton-pump inhibitors (PPIs). They also ranked these treatments from least expensive to most expensive per quality-adjusted life-year (QALY): NSAIDs plus $\mathrm{H}_{2}$ RAs; NSAIDs plus misoprostol; NSAIDs alone; coxibs; COX2 preferential inhibitors; and NSAIDs plus PPIs.
Analysis showed that NSAIDs plus $\mathrm{H}_{2} \mathrm{RAs}$ are safer and less costly than NSAIDs alone. If health-care expenditure of $£ 140,000$ per QALY is acceptable, NSAIDs plus $\mathrm{H}_{2} \mathrm{RAs}$ are the optimal treatment, but if expenditure of $>£ 140,000$ per QALY is possible, then NSAIDs plus PPIs are the optimal treatment.

The analysis was limited by the quality of available data and by assumptions made in the analytic model. Further studies should be carried out to improve the quality of information available to decision makers.

Original article Elliott RA et al. (2006) Preventing nonsteroidal anti-inflammatory drug-induced gastrointestinal toxicity: are older strategies more cost-effective in the general population? Rheumatology 45: 606-613

\section{Health literacy in patients with rheumatoid arthritis}

Effective communication between physician and patient is important, particularly for patients with chronic diseases such as rheumatoid arthritis (RA). Without being adequately informed, patients can have difficulty participating in treatment decisions, complying with therapy regimens, and monitoring their disease. Health-care providers commonly use written material to supplement verbal communication and, therefore, a minimum level of literacy is required to use the health-care system effectively. Indeed, illiteracy is strongly associated with poor health status, independent of other sociodemographic factors.

Buchbinder and colleagues carried out a trial to assess the health literacy (the ability to obtain, process and understand basic health information) of patients with $\mathrm{RA}$ in a socioeconomically advantaged suburb in Australia. The 80 patients who participated were assessed by the Test of Functional Health Literacy in Adults (TOFHLA), the Rapid Estimate of Adult Literacy in Medicine (REALM), and the Test of Reading Comprehension (TORCH). Patients were blinded to the exact purpose of the study to prevent those with poor literacy skills declining to participate. The study showed that $10 \%$ of patients had inadequate or marginal health literacy, or a reading age at or below the US equivalent of $7^{\text {th }}-8^{\text {th }}$ grade.

The authors conclude that routine assessment could identify patients with limited literacy skills, which might lead physicians to 